\title{
Comprehensive Management Platform of Natural Disasters Based on Cloud Computing
}

\author{
Xuejun Zong, Qiang Li, Zhongjun Yang, Kan He, and Dimiter Velev
}

\begin{abstract}
Aiming at the problems of the construction of the service platform for the comprehensive management of natural disasters in China, and combining the cloud computing technology, the idea of comprehensive management service platform based on cloud computing is proposed. The platform can be used as a unified service platform for the comprehensive management of natural disasters. The platform integrates the functions of natural disaster data collection and storage, risk management system, monitoring and warning system, emergency response and decision, disaster evaluation, disaster business development and customization. Realize the new integration of knowledge resources of natural disasters and provide a variety of disaster information service for all kinds of users according to the authority. Not only improve the service quality, but also reduce the cost of operation and maintenance, and effectively improve the information, digital and intelligent level of disaster management.
\end{abstract}

Index Terms-Natural disaster, comprehensive management, cloud computing.

\section{INTRODUCTION}

In recent years, under the background of global warming and world economic integration, many kinds of natural disasters, such as landslides, earthquakes, tsunamis and floods occur frequently in the global scope. The natural disasters come without warning and are impossible to avoid. As a result, natural disasters cause much serious casualties and property losses. What's worse, the damage caused by natural disasters need a very long time to recover [1], [2]. The integrated management of natural disasters is the more effective and advanced means and ways for natural disaster prevention, mitigation and management. After the reform and opening up, China has carried out a lot of useful exploration work on the risk assessment and management of natural disasters. However, compared with the advanced comprehensive integrated management platform established by United States, Japan and other developed countries, our research on the service platform for the comprehensive management of natural disasters exists some deficiencies [3], [4]: a wide range of existing natural disaster management system cause a lot of waste of resources and bring many maintenance and updates difficulties; most of the disaster management system

Manuscript received December 9, 2015; revised June 3, 2016. This work was supported in part by the Bulgarian National Science Fund for the support under the Grant № DFNI-I02/ 15 from 2014.

Xuejun Zong, Qiang Li, Zhongjun Yang, Kan He are with the Shenyang University of Chemical Technology, Shenyang, China (syictauto@163.com, syict_lq@163.com, syict_yzj@163.com,29409385@qq.com).

D. Velev is with the University of National and World Economy, Sofia 1700, Bulgaria (e-mail: dgvelev@unwe.bg). developed by C/S framework, service scope is limited; can't provide the flexibility services based on the needs of users, etc.

The vigorous development of cloud computing technology provides a solution to solve the above problems and this paper presents a preliminary idea of constructing a comprehensive management service platform of natural disasters based on cloud computing. This platform integrated disaster prevention, disaster reduction and disaster relief can realize the sharing of software and hardware resources and information. In addition, users of the platform can quickly access on demand or customize their own business. Through the use of the platform can effectively improve the scientific and effective management of natural disasters.

\section{Cloud COMPuting AND INTEGRATED MANAGEMENT OF NATURAL DISASTERS}

In order to achieve the integrated management of natural disasters on a unified platform that integrate of hardware and software resources and scheduling algorithm, the platform should have the following characteristics [5]:

1) Platform has high security and stability, convenient and friendly operation page and it can support a variety of access to Web.

2) Ability to store massive amounts of data, and can expand capacity on demand.

3) Can call a variety of computing tools for the processing of big data easily and quickly.

4) Provide various service interfaces and software and hardware resources for application development.

5) Can provide services for a variety of users, and users can be customized services.

Cloud computing is a model for enabling convenient, on-demand network access to a shared pool of configurable computing resources (e.g., networks, servers, storage, applications, and services) that can be rapidly provisioned and released with minimal management effort or service provider interaction [6]. It has large numbers of characteristics.

On-demand self-service: has the ability to provide resources and services quickly and users can expand and use cloud computing resources according to their actual needs. Users can easily apply for the computing ability through Internet and service providers can timely distribution and recovery of resources.

Broad network access: provides self-service through the Internet. Users not only do not need to deployment related complex hardware and application software, but also do not need to know the physical location of the resource and 
allocation of information. Thus, they can obtain the cloud computing resources through the Internet or internal network.

Resource pooling: allocates multiple consumers with different physical and virtual resources by using multi tenant mode dynamically and reallocates resources according to the consumer's needs. Customers can use a higher level of abstraction of cloud computing resources and usually do not need to control or know the exact location of the resources.

Rapid elasticity: deployment of resources or access to services quickly. The computing power of the service provider can quickly and flexibly implement the resource supply according to the user's demand. Cloud computing platform can quickly deploy and provide resources according to customer needs.

Measured service: Cloud service system can provide corresponding measure mode according to the type of service and cloud automatic control system optimizes resource utilization by using measurement capability of some appropriate abstract service. Moreover, it can also monitor control and management the process of resources. At the same time, it can provide a transparent service between the supplier and the service consumer.

These characteristics of cloud computing just fit the need of natural disaster management. Cloud computing provides a new thought and a powerful assistant tool for the comprehensive management of natural disasters. Build an integrated natural disaster management services platform in the cloud computing platform that integrates a variety of resources, computing, storage, networking, etc., and constitute a huge pool of virtual resources. Users of cloud services never have to buy or upgrade computing hardware and they can obtain the required resources through the browser run in computers, mobile phones and other terminals anytime. It not only improves the efficiency of resource use, but also reduces the operation and maintenance costs [7].

\section{SERVICE LEVEL OF THE PlATFORM}

Cloud computing provides a new way for the comprehensive management of natural disasters as a service-oriented computing model. The platform makes all the hardware and software resources, data, services and applications deployed in the cloud unified platform. Through the unified management of cloud computing platform, improve the efficiency and effectiveness of the management of natural disasters [8]. Service levels of the platform include: Software as a Service (SaaS), Platform as a Service (PaaS), Data as a Service (DaaS), Infrastructure as a Service (IaaS), as shown in Table I.

Software as a Service: provides users with software applications on-demand, software upgrades and maintenance. This service level provides users with natural disaster monitoring alert service, emergency command and rescue services, information services, restoration and reconstruction. Through such a unified platform, it is conducive to the establishment of a unified standard and unified standards of natural disaster management business. If the application service can't meet the needs of users, users can customize their applications in the service layer of PaaS.
TABLE I: SERVICE LEVELS OF THE PLATFORM

\begin{tabular}{cl}
\hline Service level & \multicolumn{1}{c}{ Function } \\
\hline $\begin{array}{c}\text { Software as a } \\
\text { Service (SaaS) }\end{array}$ & $\begin{array}{l}\text { Provide Internet-based } \\
\text { application services }\end{array}$ \\
Platform as a & $\begin{array}{l}\text { Provide application } \\
\text { development, deployment } \\
\text { and management services }\end{array}$ \\
Service (PaaS) & Data processing, storage \\
Data as a & and other services \\
Service (DaaS) & $\begin{array}{l}\text { Provide infrastructure } \\
\text { deployment services }\end{array}$ \\
\hline
\end{tabular}

Platform as a Service (PaaS): provides users with personalized services, including custom and development of natural disasters; according to the needs of the users can timely and dynamic expansion of resources, and thus can improve the practicality and agility of the business. Users do not need to manage or operate the underlying cloud infrastructure, including operating systems, server systems, storage systems, networks, etc.

Data as a Service (DaaS): is the basis of the whole platform, including data collection, data processing and standardization, update and maintenance, etc. Use virtualization technology to store data in the physical storage devices of the platform to ensure the validity, security and scalability of data. It can provide data services for disaster warning, risk assessment and provide users with metadata services, second development of product data services according to the authority. Furthermore, users can also build their own database on the platform based on their demand.

Infrastructure as a Service (IaaS): utilizes virtualization technology to process the physical resources, and realize the optimization of internal management and resource management. Thus it can provide users with services dynamically and flexibly. Users can also deploy and run a variety of application software based on the leased cloud infrastructure without the need to manage and control the underlying hardware infrastructure.

Cloud computing offers capabilities to automate many services, to expedite the implementation of secure configurations to information devices, to reduce dependence on removable media due to broadband services, and to lower costs in disaster recovery and data storage.

Many companies and organizations typically store their business information in multiple data systems across many different servers located in different countries around the world. Trying to track down the information that is required and then accessing through some form of networked computer system can be difficult at the best of times. This is difficult if working remotely and there is a need to connect into a business system via a laptop computer. When losing the server architecture, the time needed to rebuild everything can cost a lot. If everything is in the cloud, the organizations can restart their operations as soon as they have access to the Internet.

In cloud operations, it is expected that multiple copies of a data set will be created and kept in sync. The practice of a business offering a free service to another in an area hit by natural disaster could become a new form of international aid. 
Under some circumstances virtual machine images of existing workloads can be created in the enterprise data center and stored in a cloud data center. In the event of a failure of the former, the virtual machines serve as recovery mechanisms that can be reactivated in the cloud.

Natural disasters may severely damage Internet access and communications which make it difficult to access cloud-based servers, applications and data storage. The interruption of network availability is usually temporary, while companies that relied purely on local infrastructure may find their servers completely destroyed and their backup totally lost [9].

When a disaster occurs, telephone lines in disaster areas are overloaded with calls. Using cloud computing for the emergency management could also improve the computer database by providing government agencies with detailed, real-time disaster information. Recovering data after as disaster costs typically twice as much as replacing compromised hardware and software. In the case of cloud computing, recovery costs are considerably lower since only local computers used to access the Internet are at risk and user data and cloud servers are protected far from the disaster site. In the case of a disaster striking a cloud computing data center, user data will not be lost since suppliers of cloud infrastructure replicate user data and cloud servers across multiple data centers. If a city that uses cloud computing to manage its community development department had the misfortune to lose all its IT equipment in a hurricane or tornado, it could start the task of rebuilding the next day from any location using laptops and an Internet connection [10].

A common concern about using a cloud computing application is that data will be less secure. In practice, however, entrusting information assets to a recognized cloud computing provider generally increases the safety of those assets since on premise IT security practices are often sub-standard. Given that smaller IT departments struggle to design, fund and maintain secure systems while cloud computing providers deliver IT infrastructure as their primary business and competence, moving to cloud computing and SaaS will almost certainly increase security for the majority of IT users [10].

One of the benefits of cloud computing is that information and operations are hosted in well protected data centers Top cloud providers keep information on thousands of systems and in numerous locations. Redundancy, availability and reliability are hallmarks of cloud computing, so that users can access your information quickly, no matter where they are located. For example, Amazon and Microsoft have data centers all over the world, with tens of thousands of processing units and storage. They have miles of cables, generators and batteries to run these systems for days or even weeks in the event of power disruptions. Many are located in places less susceptible to harm from natural disasters. Some service providers build data centers under ground inside massive concrete buildings. All these precautions ensure that you have access to services and data $24 \times 7$.

The location of a data center can have a significant impact on the performance of applications running in a cloud computing environment. If a cloud computing provider's primary data center is in country that is to far away from the current location of the natural disaster, the performance of that application will be adversely affected by the prolonged time needed for the messages to travel between the data center and the users in disaster condition.

However, there are certain requirements that must be imposed on the utilization of cloud computing in emergency management [11]:

- The cloud provider must be responsible for data confidentiality, integrity and availability. This accounting should not be a cursory exercise, but one that demonstrates complete detailed controllership and accountability at each point (and each vendor they use) within the cloud. Yes, this means during access, authentication, transmission, processing, storage, recovery and destruction.

- Specifications must be prepared regarding ways how the cloud provider will preserve and produce data from requests. Depending on the compliance and legal objectives, this can extend to a few more providers within the cloud and can impact systems that are shared with other cloud clients.

- Data encryption must be considered. The geographical and logical location of its use must be taken into account, the minimum and maximum levels required, laws that may impact use, and if encryption will block the ability to monitor and track the data and threats.

- The cloud provider must possess a crisis management process that will have the appropriate technical, organizational and procedural measures. This can include financial crisis such as vendor bankruptcies, mergers, acquisitions along with traditional areas like weather, geological disruptions, epidemics, etc.

Several additional objectives can be defined regarding cloud computing application in emergency management [12]:

- Using the computing cloud to rapidly converge geographically dispersed global experts at the start of an international incident, deploy a foundation of guidance in accordance with community leaders in a manner that empowers community members through education and smart technologies to support mitigation, response, recovery, and a resumption of societal normalcy at a level of functioning an order of magnitude higher than existed before.

- Using mobile communication devices for rapid threat/damage assessment of occurring events, as well as damage to critical infrastructure inland that necessitates mass sheltering of displaced community members.

- Using the power of non-governmental organizations, rapidly responding government and corporate groups, international groups, social networking communities and other resilient networks to locate and gather information, as well as to send help.

Clouds are secure and though adaptation of authenticity, encryption, and meeting security software regulation large concern about secure can be put aside. Furthermore, the cloud is not in one place, meaning the risk of systems failures substantially decreases. In the case of cloud computing, recovery costs are considerably lower since only local computers used to access the Internet are at risk and user data and cloud servers are protected far from the disaster site. In 
the case of a disaster striking a cloud computing data center, user data will not be lost since suppliers of cloud infrastructure replicate user data and cloud servers across multiple data centers.

\section{ARChITECTURE OF THE PLATFORM}

The architecture of comprehensive management platform of natural disaster based on cloud computing is composed of four parts: infrastructure layer, management middleware layer, application development and service layer and SOA construction layer, as shown in Fig. 1.

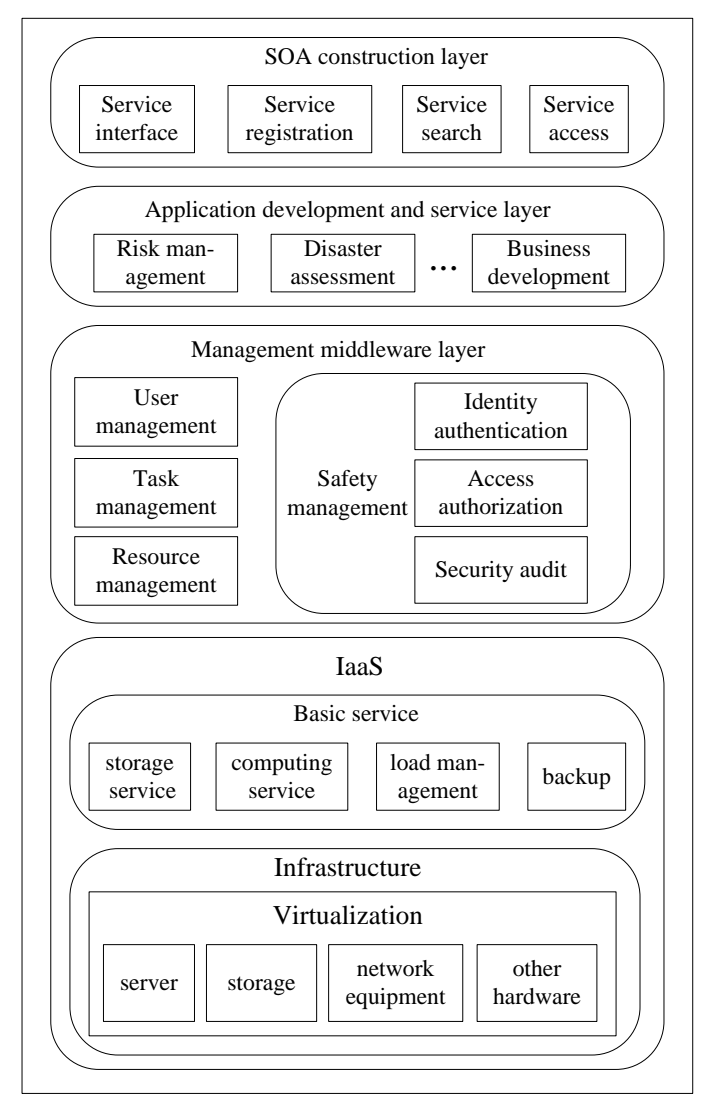

Fig. 1. Architecture of the platform.

Infrastructure layer: provides the necessary hardware resources and storage functions, and provides the necessary basic environment for the development in a unified scheduling algorithm and resource management. It's mainly to meet the user's computing requirement and storage needs and can reduce the waste of resources and cost investment from the duplication of construction.

Management middleware layer: it is main function is to ensure the safe operation of platform and monitor the basic resources of platform. As a result, it's able to realize the scheduling of tasks in a variety of applications, so that resources can be safely and effectively used to provide services.

Application development and service layer: provides a variety of research and development environment for the business development. Developers do not need to focus on high-tech hardware resource structure and can do some development that meet the needs of users by grasping the characteristics of the platform. The layer is a model for the development of software and services through the Internet and users do not need to buy, install, maintain or upgrade software.

SOA construction layer [13]: encapsulates cloud computing capabilities into standard Web service, and incorporates it into the SOA system for the management and use, including service interfaces, service registration, service discovery, service stations and other access and building services. Service-oriented architecture is a component model, which applies the different functional units of the services to define a good interface between these services.

\section{CONCLUSION}

Cloud computing has been proved to be a mature and reliable solution, which can effectively solve the problem of resource sharing. The needs and problems faced of natural disasters just coincide with the function of cloud computing. Cloud computing provides a new idea and approach for the construction of comprehensive management platform of natural disasters. Using virtualization technology, distributed storage technology, distributed computing technology and dynamic resource pool management mode of cloud computing, provide a unified service platform for the comprehensive management of natural disasters. It can achieve a new integration of knowledge resources of natural disasters and the existing various natural disaster management system through this platform. In addition, it not only improves the resource utilization efficiency, but also reduces the operation and maintenance costs. More importantly, it can provide users with more convenient and secure computing, storage, and other services.

\section{ACKNOWLEDGMENT}

The authors express their gratitude to the Bulgarian National Science Fund for the partial financial support under the Grant № DFNI-I02/15 from 12.12.2014, titled "Information System for Integrated Risk Assessment from Natural Disasters".

\section{REFERENCES}

[1] R. Arden. (Aug. 26th, 2011). Surviving a Natural Disaster with Cloud Computing. Available: http://edocumentsciences.com/

[2] C. Babcock. (2011). Cloud becoming a major disaster recovery strategy. Information Week. Available: http://mobile.informationWeek.com

[3] J. H. Wen, Z. E. Yin, and Q. J. Meng, "Mainstreaming disaster risk management in China", Progress in Geography, vol. 29, no. 7, pp. 771-777, 2010.

[4] C. J. Ni, "Discussion on the approaches of natural disaster risk assessment," Journal of Catastrophology, vol. 28, no. 2, pp. 1-5, 2013.

[5] X. A. Li, H. N. Song, Z. T. He et al., "Research on comprehensive emergency management platform based on cloud computing," Information Technology, vol. 25, no. 5, pp. 18-21, 2014.

[6] P. Mwll and T. Grance, "The NIST definition of cloud computing," National Institute of Standards and Technology, 2011.

[7] Z. Wang, "Research on deployment of application resources based on Cloud Platform," Wuhan: Huazhong University of Science and Technology, 2013.

[8] D. Velev and P. Zlateva, "A feasibility analysis of emergency management with cloud computing integration," International Journal of Innovation, Management and Technology, vol. 3, no. 2, pp. 188-193, 2012.

[9] C. Road, "The natural disaster hard to predict makes the best of the disaster backup scheme in the high in the clouds," Chip Road2011, 2011. 
[10] T. Bradley, "Embrace the cloud for natural disaster recovery," $P C$ World, 2011

[11] R. Lawhorn, "Tarantino-style approach to secure cloud computing," Sec Techno, 2010.

[12] J. Savageau. (2010). Social networking through disaster - exercise 24, 2010.

[13] H. F. Chen, B. T. Sun, and X. Z. Chen, "Research on China earthquake loss estimation system based on cloud computing," Journal of Earthquake Engineering and Engineering Vibration, vol. 33, no. 1, pp. 198-203, 2013

Xuejun Zong is Professor in the Shenyang University of Chemical Technology, Shenyang, China. He graduated the Department of Automation of the Shenyang University of Chemical Technology in 1991. His main areas of academic and research interest are system integration, advanced process control, predictive control, optimation control, fault diagnosis.

Qiang Li is Lecturer in the Shenyang University of Chemical Technology, Shenyang, China. His main areas of academic and research interest are computer applications, system integration.

Zhongjun Yang is Lecturer in the Shenyang University of Chemical Technology, Shenyang, China. He holds an M.Sc. degree in the Department of Information Engineering of the Shenyang University of Chemical Technology. His main areas of academic and research interest are Research of Adaptive monitoring and fault diagnosis method of complex industrial production process; computer information integration, optimation control, fault diagnosis.

He Kan is Lecturer in the Shenyang University of Chemical Technology, Shenyang, China. He holds M.Sc. degree in the Department of Information Engineering of the Shenyang University of Chemical Technology. His main areas of academic and research interest are computer applications, database development, WEB design.

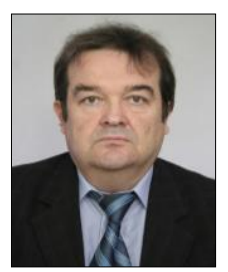

Dimiter Velev is Professor in the Department of Information Technologies and Communications at the University of National and World Economy, Sofia, Bulgaria. He holds an M.Sc. degree in Electroengineering from the Sofia Technical University, Bulgaria and Ph.D. degree in engineering sciences from the Institute of Modeling Problems in Power Engineering at the National Academy of Sciences of Ukraine, Kiev, Ukraine. His main areas of academic and research interest are internet-based business systems modeling and development, service oriented architectures, online social networks, cloud computing, web applications development and programming. 